\title{
BMJ Open Steroids In caRdiac Surgery (SIRS) trial: acute kidney injury substudy protocol of an international randomised controlled trial
}

Amit X Garg, ${ }^{1}$ Jessica Vincent, ${ }^{2}$ Meaghan Cuerden, ${ }^{3}$ Chirag Parikh, ${ }^{4}$ P J Devereaux, ${ }^{1}$ Kevin Teoh, ${ }^{1}$ Salim Yusuf, ${ }^{1}$ Ainslie Hildebrand, ${ }^{5}$ Andre Lamy, ${ }^{1}$ Yunxia Zuo, ${ }^{6}$ Daniel I Sessler, ${ }^{5}$ Pallav Shah, ${ }^{7}$ Seyed Hesameddin Abbasi, ${ }^{8}$ Mackenzie Quantz, ${ }^{1}$ Jean-Pierre Yared, ${ }^{5}$ Nicolas Noiseux, ${ }^{9}$ Georgios Tagarakis, ${ }^{10}$ Antoine Rochon, ${ }^{11}$ Janice Pogue, ${ }^{1}$ Michael Walsh, ${ }^{1}$ Matthew T V Chan, ${ }^{12}$ Francois Lamontagne, ${ }^{13}$ Abbas Salehiomran, ${ }^{8}$ Richard Whitlock, ${ }^{1}$ on behalf of the SIRS Investigators

To cite: Garg AX, Vincent J, Cuerden M, et al. Steroids In caRdiac Surgery (SIRS) trial: acute kidney injury substudy protocol of an international randomised controlled trial. BMJ Open 2014;4:e004842. doi:10.1136/bmjopen-2014004842

- Prepublication history for this paper is available online. To view these files please visit the journal online (http://dx.doi.org/10.1136/ bmjopen-2014-004842).

Received 11 January 2014 Accepted 30 January 2014

CrossMark

For numbered affiliations see end of article.

Correspondence to Dr Amit Garg; amit.garg@|hsc.on.ca

\section{ABSTRACT}

Introduction: Steroids In caRdiac Surgery trial (SIRS) is a large international randomised controlled trial of methylprednisolone or placebo in patients undergoing cardiac surgery with the use of a cardiopulmonary bypass pump. At the time of surgery, compared with placebo, methylprednisolone divided into two intravenous doses of $250 \mathrm{mg}$ each may reduce the risk of postoperative acute kidney injury (AKI).

Methods and analysis: With respect to the study schedule, over 7000 substudy eligible patients from 81 centres in 18 countries were randomised in December 2013. The authors will use a logistic regression to estimate the adjusted OR of methylprednisolone versus placebo on the primary outcome of AKI in the 14 days following surgery (a postoperative increase in serum creatinine of $\geq 50 \%$, or $\geq 26.5 \mu \mathrm{mol} / \mathrm{L}$, from the preoperative value). The stage of AKI will also be considered, as will the outcome of AKI in those with and without preoperative chronic kidney disease. After receipt of grant funding, the authors began to record additional perioperative serum creatinine measurements in consecutive patients enrolled at substudy participating centres, and patients were invited to enroll in a 6-month serum creatinine collection. In these trial subpopulations, the authors will consider the outcome of AKI defined in alternate ways, and the outcome of a 6 -month change in kidney function from the preoperative value.

Ethics and dissemination: The authors were competitively awarded a grant from the Canadian Institutes of Health Research for this SIRS AKI substudy. Ethics approval was obtained for additional serum creatinine recordings in consecutive patients enrolled at participating centres. The additional kidney data collection first began for patients enrolled after 1 March 2012. In patients who provided consent, the last 6-month kidney outcome data will be

\section{Strengths and limitations of this study}

- International recruitment across 81 centres in 18 countries will provide generalisable estimates of the treatment effect.

- The kidney data collection schedule in this large international trial is feasible and efficient.

- Additional studies of the effects of methylprednisolone in cardiac surgery can consider multiple measures of kidney function over time, before and far after acute kidney injury, examine trajectories of kidney function loss and new markers of kidney function or injury.

collected in 2014. The results will be reported no later than 2015.

Clinical Trial Registration: Number NCT00427388.

\section{BACKGROUND}

Worldwide, about two million cardiac surgeries are performed each year, and most surgeries use a cardiopulmonary bypass pump. About one-third of patients undergoing such procedures develop acute kidney injury (AKI) (defined by an acute rise in serum creatinine of $50 \%$ or more or an acute rise of $26 \mu \mathrm{mol} / \mathrm{L}$ or more), and $1 \%$ receive acute dialysis. This means there are several hundreds of thousands of cases of AKI attributed to cardiac surgery worldwide each year with about 20000 cases receiving acute dialysis. When AKI occurs (compared with when it does not), it associates with increased mortality, a longer hospital length of stay and higher healthcare costs. ${ }^{1-3}$ 
The major mechanism of AKI in cardiac surgery is impaired kidney perfusion causing an initial hypoxic insult followed by activation of inflammatory pathways during reperfusion (schematic depicted in figure 1). ${ }^{4}$ In animal models of kidney ischaemia-reperfusion injury, an elaboration of proinflammatory cytokines, reactive oxygen species and interstitial inflammation all result in AKI. ${ }^{4}$

In this setting, an inexpensive drug that attenuates the inflammatory response and related consequences could have a worldwide impact. Corticosteroids are an inexpensive, generic class of anti-inflammatory agents. We provide four lines of evidence to support the hypothesis that they reduce the risk of AKI from cardiac surgery with cardiopulmonary bypass. ${ }^{4-7}$ The mechanisms for AKI prevention are presented in figure 1.

First, corticosteroid use may mitigate perioperative cardiac events. This may occur through a few processes including the inhibition of cytokines released during cardiopulmonary bypass (figure 1). Many studies of corticosteroid use report postoperative improvements in the cardiac index and mean arterial pressure, with less use of inotropic support. A meta-analysis demonstrates that perioperative corticosteroid use clearly reduces the risk of postoperative atrial fibrillation (44 studies). ${ }^{8}$ Acute cardiac events are inextricably linked to AKI events; both events often co-occur in the non-operative setting. The process may include hypotension as a primary inciting event or from the cardiac injury itself, or from the aggressive use of diuretics used to treat acute heart failure.
Second, corticosteroid use targets the key pathological mechanisms for the development of AKI (displayed in figure 1 along with where steroids act to prevent AKI). This involves reducing the systemic inflammatory response syndrome from cardiopulmonary bypass surgery, with reductions in inflammatory mediators, cytokines, complement, transcription factors and adhesion molecules.

Third, in the largest multicentre randomised trial of dexamethasone in cardiac surgery with a cardiopulmonary bypass pump, 4494 adult patients from the Netherlands were randomly assigned to receive dexamethasone $(1 \mathrm{mg} / \mathrm{kg}$ of body weight with a $100 \mathrm{~kg}$ maximum) or placebo administered as a single intravenous injection after the induction of anaesthesia before the initiation of cardiopulmonary bypass. ${ }^{7}$ An outcome of acute renal failure defined by the study investigators was an increase in postoperative serum creatinine of at least three times the preoperative value, or a serum creatinine more than $350 \mu \mathrm{mol} / \mathrm{L}$ associated with an acute increase of serum creatinine of at least $44 \mu \mathrm{mol} / \mathrm{L}$, within 30 days of surgery. This outcome of acute renal failure was one of five components of the primary composite outcome. Dexamethasone versus placebo resulted in trends in the reduction of the primary outcome and the acute renal failure outcome that were non-significant (primary outcome: 157 patients of 2235 with dexamethasone $(7.0 \%)$ vs 191 patients of 2247 with placebo $(8.5 \%)$; relative risk reduction $17 \%(95 \%$ CI $-1 \%$ to $33 \%$ ); acute renal failure outcome 28 patients of 2235
Figure 1 Inflammation in cardiac surgery with a cardiopulmonary bypass pump, and mechanism by which acute kidney injury may be prevented by perioperative corticosteroids. Figure modified from Rosner and Okusa, ${ }^{4}$ and reproduced with permission.

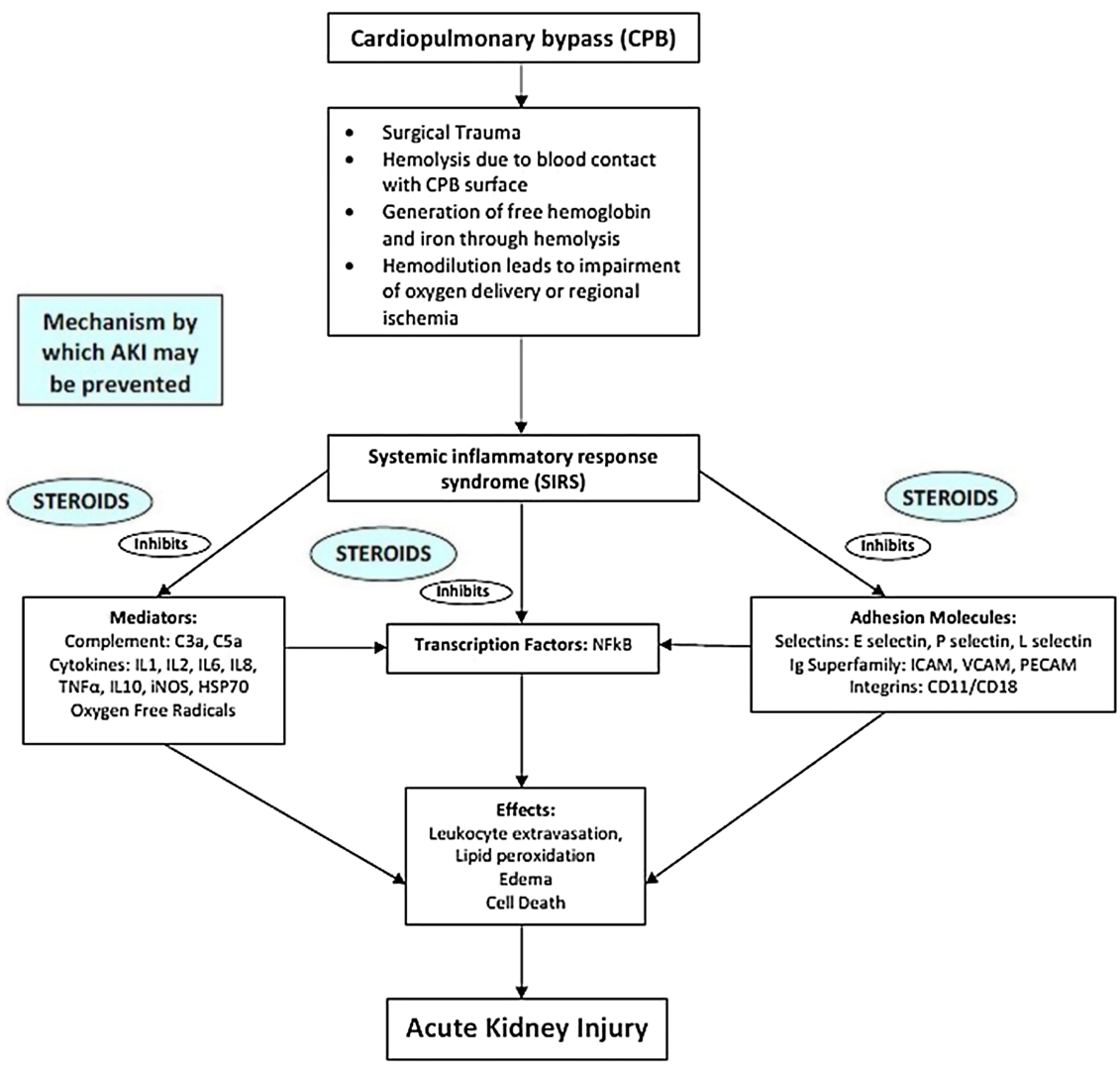


with dexamethasone (1.3\%) vs 40 patients of 2247 with placebo $(1.8 \%)$, relative risk reduction $30 \%(95 \%$ CI $-14 \%$ to $56 \%)$.

Two of the four existing small randomised controlled trials raise the possibility that corticosteroids may prevent AKI in cardiopulmonary surgery. In one trial, 29 children were randomised to receive either intravenous dexamethasone $(1 \mathrm{mg} / \mathrm{kg})$ or saline $1 \mathrm{~h}$ prior to cardiopulmonary bypass surgery. ${ }^{5}$ In the dexamethasone group, 1 of 15 (7\%) children demonstrated AKI versus 7 of $14(50 \%)$ children in the saline group; $\mathrm{p}=0.014$ (AKI defined by $\geq 18 \mu \mathrm{mol} / \mathrm{L}$ increase in serum creatinine compared with the preoperative value). Another trial randomised 236 adults undergoing elective valvular or coronary artery bypass surgery to intravenous dexamethasone $(0.6 \mathrm{mg} / \mathrm{kg})$ or placebo soon after the induction of anaesthesia. ${ }^{6}$ Three of $110(2.7 \%)$ patients with placebo versus none of the 110 patients with dexamethasone developed AKI (where AKI was defined by use of haemodialysis or oliguria). The results were statistically non-significant, recognising there were only three events, $p=0.24$. A non-statistically significant reduction in the risk of AKI was observed in the other two randomised trials (enrolled 86 and 20 patients, respectively, with the former also limited by a small number of events (2 episodes of AKI (undefined), 1/43 in steroid group versus $1 / 43$ in placebo group) ). ${ }^{9} 10$

Fourth, intravenous corticosteroids are a mainstay of therapy for many acute processes that involve kidney inflammation: acute glomerulonephritis, vasculitis, lupus and resistant forms of acute allergic interstitial nephritis. It is rational to hypothesise similar benefits in cardiopulmonary bypass.

We are conducting the Steroids In caRdiac Surgery trial (acronym SIRS). The rational and design of this large, international, randomised placebo-controlled trial are described elsewhere. ${ }^{11}$ In brief, adults at high risk of postoperative complications who are undergoing cardiac surgery with a cardiopulmonary bypass pump are randomly allocated to receive either methylprednisolone divided into two intravenous $250 \mathrm{mg}$ doses (one dose at the start of anaesthesia and the other at the start of the cardiopulmonary bypass pump) or matching placebo. Allocation is performed by a central randomisation service and is stratified by centre. Patients, healthcare providers, data collectors and outcome adjudicators are blinded to treatment allocation. This trial is funded by the Canadian Institutes of Health Research. With respect to the study schedule, over 7500 patients (from the last in the order of registration) from 82 centres in 19 countries were randomised on 20 December 2013. The primary outcome is 30 day all-cause mortality. The most important secondary outcomes are a 30-day composite outcome of death, myocardial infarction, stroke, renal failure and respiratory failure. Renal failure is defined using thresholds of stage 3 AKI described in recent guidelines. ${ }^{12}$ Secondary safety outcomes include postoperative infection, wound complications, gastrointestinal haemorrhage, delirium and insulin use.
In addition to the primary outcome, SIRS is uniquely positioned to determine the effects of corticosteroids on various stages and alternate definitions of AKI (and whether the treatment effect is homogeneous across these stages), to consider whether the treatment effect differs in those with and without preoperative chronic kidney disease (CKD), and to investigate the effect of corticosteroids at the time of surgery on kidney function 6 months later. We were competitively awarded an additional grant from the Canadian Institutes of Health Research to examine these issues in a SIRS AKI substudy. The questions detailed in this grant are presented below followed by a prespecified analytical plan.

\section{Primary questions}

1. In patients undergoing cardiac surgery with a cardiopulmonary bypass pump, does the use of intravenous methylprednisolone at the time of surgery compared with placebo alter the risk of postoperative AKI? Is the treatment effect (the observed relative risk reduction) statistically homogeneous across multiple alternate stages and definitions of AKI? Hypothesis: Corticosteroids reduce the risk of postoperative AKI, with a similar treatment effect across alternate definitions of AKI.

\section{Secondary questions}

2. Does the presence of preoperative CKD modify the effect of treatment (methylprednisolone versus placebo) on AKI? Hypothesis: A greater relative risk reduction will be observed in patients with preoperative CKD than those without CKD (as we demonstrated in another setting-a large international randomised trial of coronary artery bypass surgery performed either with or without a bypass pump).

3. Does methylprednisolone versus placebo at the time of surgery alter kidney function 6 months after surgery? Hypothesis: We will be unable to demonstrate that corticosteroids improve this outcome (due to either the study methods or the limited impact of any observed AKI risk reduction on longer term kidney function).

\section{KIDNEY DATA COLLECTION AND ANALYTIC PLAN}

SIRS enrollment began in 2007. To refine the analytical plan, we reviewed SIRS data as of November 2013 (without knowledge of the treatment allocation and recognising SIRS data is actively being adjudicated and data discrepancies reconciled).

\section{SIRS kidney data collection}

\section{Kidney data collection in all SIRS patients}

A preoperative serum creatinine is measured and recorded in the 30 days prior to surgery. The date of surgery (and not the date of randomisation) will be used to identify the start of follow-up in this protocol; the median (IQR) number of days between the date of 
randomisation and the date of surgery is $1(0-1)$, and the $<2 \%$ of randomised patients expected not to receive surgery will be excluded from the analysis (see Patient selection section). A single peak serum creatinine in the 14 days following surgery is recorded along with the date of this measurement. At the start of our study, 2 weeks was felt to be a reasonable period in which any AKI related to the surgery would manifest (either from the initial insult during surgery, and/or related to a second insult that occurred soon after surgery). Since the launch of SIRS, international AKI guidelines were published in 2012 which define AKI by an increase in serum creatinine of $\geq 26.5 \mu \mathrm{mol} / \mathrm{L}$ within $48 \mathrm{~h}$, or an increase in serum creatinine of $\geq 50 \%$ known or presumed to have occurred within 7 days. ${ }^{12}$ Currently, for all SIRS participants, the single peak postoperative serum creatinine is recorded a median of 2 days after surgery (IQR $1-4$ days). Approximately $60 \%$ of patients have this peak measurement recorded within 2 days of surgery, and $70 \%, 80 \%$ and $83 \%$ of patients have this measurement recorded within 3,4 and 5 days of surgery, respectively. Less than $1.5 \%$ of patients die in the operating room or in the $48 \mathrm{~h}$ after surgery (which may influence serum creatinine measurement). Less than $2 \%$ of patients are expected to have a missing peak serum creatinine value within 14 days of surgery, and in such cases in the absence of receipt of acute dialysis, we will carry forward the prerandomisation serum creatinine value for all analyses (which means the patient will not have developed AKI; see Analysis section). No urine output data have been collected in SIRS, given difficulties with its accurate measurement in the setting of international data collection. Receipt of new dialysis for kidney failure is recorded at hospital discharge and at 30 days after surgery.

\section{Additional perioperative kidney data recordings in consecutive patients}

After receipt of grant funding for this substudy, additional perioperative serum creatinine data began to be recorded in consecutive patients enrolled at substudy participating centres (earliest centre to start this process began in March 2012). The data collection includes all serum creatinine values performed as a part of routine care (and the time of measurement) within the 14 days after surgery. If a patient is still in hospital beyond 14 days, the most recent serum creatinine value performed in routine care prior to the hospital discharge is also recorded.

\section{6-month kidney function data}

After receipt of grant funding, in substudy participating centres, patients have been invited to enroll at the time of randomisation to record a single 6-month serum creatinine value (with instructions that a value is acceptable provided it is collected anytime between 3 and 8 months after surgery when a patient is not acutely ill).
The additional serum creatinine collection (particularly the 6-month creatinine measurement) was added to the patient's consent requests and ethical approval was received at each of the AKI substudy participating sites.

\section{Patient selection}

All SIRS randomised patients will be included in the AKI substudy except for the following three reasons: (1) those with end-stage renal disease prior to randomisation (expected $<2 \%$ of patients), as the assessment of AKI is no longer relevant (estimated-glomerular filtration rate (eGFR) $<15 \mathrm{~mL} / \mathrm{min} / 1.73 \mathrm{~m}^{2}$ as determined by the chronic kidney disease-epidemiology collaboration (CKD-EPI) equation, ${ }^{13}$ receipt of chronic dialysis or a prior kidney transplant or a baseline serum creatinine $>327 \mu \mathrm{mol} / \mathrm{L}$; the last exclusion also enables retained patients to have their new onset AKI staged according to most recent guidelines) ${ }^{12}$; (2) those missing a prerandomisation serum creatinine value, or missing age or sex (expected in $<1 \%$ of patients) as this is needed to define AKI or baseline CKD and (3) those who never underwent cardiac surgery (expected in $<2 \%$ of patients) as they will not have the opportunity to have any postoperative serum creatinine measurements. When we publish the final results, these exclusions will be reported separately in each of the methylprednisolone and placebo groups. We expect over 7000 SIRS patients will be eligible for the AKI substudy from 81 centres in 18 countries.

\section{Intention to treat}

The intention-to-treat principle will guide all primary analyses, irrespective of whether there is a deviation from the randomly allocated therapy or not (methylprednisolone or matching placebo). Currently, approximately $3 \%$ of patients did not receive the study medication, $2.4 \%$ of patients received non-study corticosteroids in the operating room and $4.4 \%$ of patients received non-study corticosteroids after surgery. At the time of final analysis, these results will be reported by treatment group.

\section{Primary definition of AKI}

We will use a mixed effects logistic regression model to obtain an estimate of the OR of AKI comparing methylprednisolone with placebo (after testing model assumptions). While logistic regression models will be used for hypothesis testing, at the time of final analysis, we will preferentially report relative risk reductions of methylprednisolone versus placebo as this metric is easier to interpret by a clinical audience (and hence we use relative risk reduction nomenclature throughout the protocol appreciating the logistic regression model produces an OR; the method to derive a relative risk reduction from an OR is described elsewhere $\left.{ }^{14}\right)$. In SIRS, this AKI outcome will use the most recent consensus criteria and will be defined as any of the following two criteria ${ }^{12}:(1)$ $\geq 50 \%$ change in the postoperative serum creatinine value from the preoperative value (( peak postoperative 
serum creatinine-preoperative serum creatinine)/preoperative serum creatinine) $\times 100$ ), (2) $\geq 26.5 \mu \mathrm{mol} / \mathrm{L}$ increase in serum creatinine after surgery from the preoperative value. Given the nature of SIRS data collection, this peak postoperative serum creatinine value is assessed in the 14 days following surgery (recognising greater than $80 \%$ of peak values are currently evident within 5 days of surgery), thus the timing in this SIRS AKI definition is slightly modified than what is presented in the AKI consensus definition. ${ }^{12}$ For the primary analysis, we will treat centre as a random effect, and will adjust for the following baseline characteristics: age (per year), sex, left ventricular function ( $\geq 50 \%, 35-49 \%, 20-34 \%,<20 \%)$, diabetes, prerandomisation ACE inhibitor or angiotensin receptor blocker use, prerandomisation statin use, prerandomisation diuretic use, preoperative eGFR category ( $\geq 60$ and $<60 \mathrm{~mL} / \mathrm{min} / 1.73 \mathrm{~m}^{2}$ ), surgery type (coronary artery bypass grafting (CABG), valve, CABG and valve) and evidence of non-elective surgery (defined by either preoperative use of inotropes or vasopressors, preoperative use of an intra-aortic balloon pump or ventricular assist device or history of a myocardial infarction within the 30 days prior to surgery). In patients who underwent surgery but are missing a postoperative serum creatinine value (expected in $<2 \%$ of patients), we will carry the prerandomisation serum creatinine value forward which should provide a more conservative estimate of the intervention effect than the alternative of removing such patients. For patients missing a left ventricular ejection fraction measurement (at present $<1.5 \%$ missing), we will impute a value of $50 \%$. If required, for each remaining covariate, we will include a missing data indicator variable (at present $<0.1 \%$ data are missing for each variable). ${ }^{15}$ We will report the $95 \%$ CI for the adjusted RRR, and a two-tailed $p$ value of $\leq 0.05$ will be considered statistically significant. We will have over $90 \%$ power to detect at least a $10 \%$ RRR in AKI with methylprednisolone versus placebo should it exist (anticipated incidence of AKI in the placebo group in SIRS, which has enrolled a group at high risk of postoperative complications, is about $38 \%$, two-tailed $\alpha 0.05, \chi^{2}$ test; table 1$)$. For completeness in the setting of a significant adjusted RRR, we will also report the unadjusted RRR (with 95\% CI) and the unadjusted absolute risk difference (with $95 \% \mathrm{CI}$ ). A significant unadjusted absolute risk difference will also be expressed as the 'number needed to treat (NNT)' (1/ absolute risk difference; a measure which indicates how many patients need to receive methylprednisolone to prevent one patient from developing AKI who otherwise would develop AKI if they receive placebo; a lower number indicating a greater benefit of methylprednisolone). The $95 \%$ CI of the NNT is the inverse of the Wald CI for the absolute risk difference. ${ }^{16} 17$

\section{Alternate definitions of AKI}

To determine how robust the AKI results are, we will examine the effect of methylprednisolone versus placebo on alternate definitions of AKI, including definitions examined in subpopulations of SIRS patients with additional serum creatinine measurements. A p value $\leq 0.05$ will be considered statistically significant in all these analyses if results are concordant with the primary SIRS AKI definition. The statistical power to detect a 20\% RRR in each of these outcomes (for all SIRS patients) is presented in table 1, and for a subpopulation with multiple postoperative serum creatinine values in table 2. As seen there is inadequate statistical power to detect a clinically important difference in some outcomes (such as the outcome of receipt of acute dialysis); however, these outcomes will be reported given their clinical significance and we will visually compare the point estimates and 95\% CI of the RRR for each outcome. As well, in an additional analysis, we will test for statistical homogeneity of the RRR across the various alternate definitions of AKI. ${ }^{18}$

\section{In all SIRS patients}

- Stage 2 AKI or more (aSIRS definition): defined by any of the following three criteria: (1) postoperative per cent change in serum creatinine $\geq 100 \%$, (2) increase

Table 1 SIRS patients meeting definitions of acute kidney injury using peak serum creatinine in the 14 days following surgery

\begin{tabular}{|c|c|c|c|c|}
\hline & $\begin{array}{l}\text { All patients } \\
(n=6598)^{\star}\end{array}$ & $\begin{array}{l}\text { Patients with a } \\
\text { preoperative eGFR } \\
\geq 60 \mathrm{~mL} / \mathrm{min} / 1.73 \mathrm{~m}^{2} \\
(\mathrm{n}=4668)\end{array}$ & $\begin{array}{l}\text { Patients with a } \\
\text { preoperative eGFR } \\
<60 \mathrm{~mL} / \mathrm{min} / 1.73 \mathrm{~m}^{2} \\
(\mathrm{n}=1930)\end{array}$ & $\begin{array}{l}\text { Statistical power } \\
\text { to detect a } 20 \% \text { relative } \\
\text { risk reduction } \\
\text { (7000 patients) (\%)† }\end{array}$ \\
\hline \multicolumn{5}{|l|}{ Primary definition } \\
\hline AKI (a SIRS definition) & 2557 (38.8\%) & $1678(36.0 \%)$ & $879(45.5 \%)$ & 99 \\
\hline \multicolumn{5}{|l|}{ Alternate definitions } \\
\hline AKI stage 2 or more (a SIRS definition) & $614(9.3 \%)$ & $395(8.5 \%)$ & $219(11.3 \%)$ & 79 \\
\hline AKI stage 3 or more (a SIRS definition) & $265(4.0 \%)$ & $132(2.8 \%)$ & $133(6.9 \%)$ & 44 \\
\hline Receipt of acute dialysis within 30 days & $164(2.5 \%)$ & $71(1.5 \%)$ & $93(4.8 \%)$ & 29 \\
\hline AKI (a SIRS definition) or death & $2625(39.8 \%)$ & $1721(36.9 \%)$ & $904(46.8 \%)$ & 99 \\
\hline
\end{tabular}


Table 2 Subpopulation of SIRS patients meeting definitions of acute kidney injury using multiple postoperative creatinine measurements

\begin{tabular}{|c|c|c|c|c|}
\hline & All patients $(n=4231)^{\star}$ & $\begin{array}{l}\text { Patients with a } \\
\text { preoperative eGFR } \\
\geq 60 \mathrm{~mL} / \mathrm{min} / 1.73 \mathrm{~m}^{2} \\
(\mathrm{n}=3016)\end{array}$ & $\begin{array}{l}\text { Patients with a } \\
\text { preoperative eGFR } \\
<60 \mathrm{~mL} / \mathrm{min} / 1.73 \mathrm{~m}^{2} \\
(\mathrm{n}=1215)\end{array}$ & $\begin{array}{l}\text { Statistical power to } \\
\text { detect a } 20 \% \text { relative } \\
\text { risk reduction } \\
\text { (4200 patients) } \dagger\end{array}$ \\
\hline AKI (a guideline definition) & $1386(32.8 \%)$ & 935 (31.0\%) & $451(37.1 \%)$ & 99 \\
\hline $\mathrm{AKI}$ for at least 2 days & $903(21.3 \%)$ & $549(18.2 \%)$ & $354(29.1 \%)$ & 94 \\
\hline AKI for at least 3 days & $575(13.6 \%)$ & $324(10.7 \%)$ & $251(20.7 \%)$ & 78 \\
\hline
\end{tabular}

in postoperative serum creatinine to an absolute value $\geq 353.6 \mu \mathrm{mol} / \mathrm{L}$ or (3) receipt of acute dialysis. This outcome uses the peak serum creatinine recorded within 14 days of surgery, or receipt of acute dialysis within 30 days of surgery.

- Stage 3 AKI (aSIRS definition): defined by any of the following three criteria: (1) postoperative per cent change in serum creatinine $\geq 200 \%$, (2) increase in postoperative serum creatinine to an absolute value $\geq 353.6 \mu \mathrm{mol} / \mathrm{L}$ or (3) receipt of acute dialysis. This outcome uses the peak serum creatinine recorded within 14 days of surgery, or receipt of acute dialysis within 30 days of surgery. This is the definition of renal failure that will be reported in the overall (non-renal SIRS) report, where renal failure is a component of a composite key secondary outcome in the trial (this composite aligns with the reporting of another prominent trial in the field). ${ }^{7}$

- Receipt of acute dialysis: receipt of acute dialysis within 30 days of surgery.

- Primary definition of AKI (aSIRS definition) or death. A composite outcome of either the primary AKI definition or death within $48 \mathrm{~h}$ of surgery. This is to account for the potential impact early deaths may have on the ascertainment of AKI.

In addition to dichotomous outcomes, we will use a linear regression model to compare the groups in the outcome of per cent change in serum creatinine ((peak postoperative serum creatinine (within 14 days of surgery) -preoperative serum creatinine)/preoperative serum creatinine) $\times 100$, adjusting for the variables described in the primary outcome analysis (assuming model assumptions are not violated ${ }^{19}$ ). We will report the result as the average difference in per cent change in serum creatinine between the two treatment groups with $95 \%$ CI.

\section{In subpopulation of SIRS patients with multiple} postoperative serum creatinine recordings

We expect that over 4200 patients (a subpopulation of SIRS patients) will be consecutively enrolled at each participating centre in the protocol to record multiple postoperative serum creatinine measurements. The AKI outcomes to be examined in this subpopulation are presented in table 2, and are:
- AKI (a guideline definition ${ }^{12}$ ): defined as any of the following two criteria: (1) an increase in serum creatinine $\geq 26.5 \mu \mathrm{mol} / \mathrm{L}$ within $48 \mathrm{~h}$ of surgery or (2) increase in serum creatinine $\geq 50 \%$ from baseline within 7 days of surgery.

- AKI for at least 2 days (or at least 3 days): defined by evidence of either postoperative increase in serum creatinine $\geq 50 \%$ from the preoperative value, or a serum creatinine $\geq 26.5 \mu \mathrm{mol} / \mathrm{L}$ from the preoperative value, evident on at least two separate days (or at least three separate days) within the 7 days of surgery. While the magnitude of the peak change in serum creatinine defines the stage of AKI in recent guidelines, a longer duration of AKI is also associated with poorer outcomes. $^{20}$

\section{Subgroup analyses: presence of preoperative CKD}

To statistically test whether the effect of treatment (methylprednisolone vs. placebo) on our primary definition of AKI differs in those with and without CKD, we will use an interaction term in a mixed effects logistic regression model where centre is treated as a random effect (assuming model assumptions are not violated). With 7000 patients, we will have over $80 \%$ power to detect a 25\% lower OR of AKI with treatment for patients with and without CKD (an estimate derived from our simulations; in another recent large trial of CABG surgery performed with and without a bypass pump, the observed per cent decrease in the OR of AKI was $40 \%$ lower in patients with CKD compared with those without CKD ( $p$ value for interaction 0.01)).

\section{Additional analyses}

Kidney function loss 6 months after surgery

This analysis will focus on those patients who at the time of surgery consent to the collection of a single 6-month serum creatinine measurement. For our main analysis, we will compare the absolute change in eGFR between the methylprednisolone and placebo groups, reporting the mean and $95 \%$ CI. We will carry forward the prerandomisation serum creatinine value for any patient missing the longer term serum creatinine measurement after surgery (for reasons of death or missing measurement) and will impute an eGFR value of $5 \mathrm{~mL} / \mathrm{min} /$ 
$1.73 \mathrm{~m}^{2}$ for the long-term measurement for any patient who developed end-stage renal disease ( $\geq 3$ months of continuous dialysis), or who died shortly after receipt of acute dialysis for severe AKI. We expect that over 800 patients will consent to participate in this longer term creatinine measurement. A sample of 800 patients will allow a minimum $10 \mathrm{~mL} / \mathrm{min} / 1.73 \mathrm{~m}^{2}$ or more difference to be detected between the groups, with over $80 \%$ statistical power (two-tailed $\alpha 0.05$, independent samples $t$ test, expected SD 50). Even in the scenario where we demonstrate corticosteroids versus placebo reduces the risk of postoperative AKI, we do not expect to demonstrate that perioperative corticosteroids versus placebo alters this 6-month outcome (which may relate to our study methods or the limited impact of the AKI risk reduction on longer term kidney function).

\section{Postoperative cardiac events}

Acute cardiac events are inextricably linked to AKI events and both events often co-occur in the nonoperative setting. In the setting where methylprednisolone reduces the risk of AKI, we will also examine cardiac events and report how frequently both cooccur.

\section{Postoperative infection events}

If we observe that methylprednisolone versus placebo increases the risk of AKI, then we examine infection events and report how frequently both cooccur.

\section{RECOGNISED LIMITATIONS}

This protocol has some limitations.

\section{Postoperative serum creatinine collection}

In SIRS, postoperative in-hospital serum creatinine measurements are recorded as performed in routine care in each participating site (in the subpopulation with serial serum creatinine measurements the median (IQR) number of measurements is $5(3-7))$. Most patients routinely have their serum creatinine measured as a part of routine care for many days after cardiac surgery, so the potential of ascertainment bias is low (ie, where methylprednisolone versus placebo alters the incidence of another event such as myocardial infarction or infection, which in turn influences the likelihood of serum creatinine measurement). At the time of final analysis, we will examine the number of measurements by treatment group, and also look at the length of hospital stay in each group to consider whether there is the potential for differential ascertainment of AKI in the two treatment groups.

\section{Errors with serum creatinine as a measure of kidney function}

In SIRS, the preoperative serum creatinine is only recorded once in the 30 days prior to cardiac surgery (where patients may be undergoing elective, urgent or emergent surgery). It would be preferably to have at least two baseline serum creatinine values on all SIRS patients, separated by at least 3 months, to more accurately define the presence of CKD. Also, in SIRS, there is no knowledge of potential serum creatinine changes close to the time of surgery (particularly in the case of emergency surgery, poor perfusion or recent contrast) which may mean that the preoperative serum creatinine value is not in a steady state (although this characteristic should be equally balanced in the methylprednisolone and placebo groups). All of these considerations increase 'noise' related to the baseline serum creatinine measurement, and reduce the ability to detect postoperative changes in the serum creatinine. To partly address this concern, we are adjusting for a definition of urgent surgery in the primary statistical models, and can also repeat the analysis excluding patients who meet the definition of urgent surgery.

Worldwide, over the last decade, there have been efforts to better standardise the serum creatinine assay. It seems likely (although not documented in SIRS) that most preoperative and postoperative serum creatinine measurements prior to hospital discharge would be performed in the same laboratory, and close in time to one another. This reduces concerns about interlaboratory and intralaboratory variability (drift) in the serum creatinine measurement. However, the same cannot be said for the 6-month measurement. Serial measures of serum creatinine assayed at a central laboratory 6 months after surgery (and longer) were desired, but were not possible to obtain in SIRS. Also, SIRS has no measures of baseline or follow-up proteinuria, which are now featured in more recent CKD staging systems.

If corticosteroids decrease creatinine production, this in theory could blunt any rise in serum creatinine after surgery and result in less patients meeting our definitions of AKI. However, in other settings such as rheumatoid arthritis, high-dose intravenous glucocorticoid (equivalent to $1000 \mathrm{mg}$ of methylprednisolone) did not reduce the amount of creatinine in subsequent urine measurements (which would have declined if creatinine production had declined). ${ }^{21}$

\section{Limited statistical power for the most clinically important kidney outcomes}

Stage 3 AKI with receipt of dialysis and long-term endstage renal disease are the most clinically important kidney outcomes. However, there will be too few events for adequate statistical power to reliably determine the effect of our intervention on these outcomes. We focus on mild-to-moderate AKI (defined by accepted thresholds of changes in serum creatinine) ${ }^{12}$ While mildto-moderate AKI is the outcome used in virtually all AKI prevention trials, we recognise that it is a surrogate outcome that may not directly impact how a patient feels, functions or survives. Similarly, the longer term outcome of between-group difference in change in eGFR is also a surrogate outcome, and concerns about its validity as a measure of kidney disease progression 
have been raised. ${ }^{22}$ For this reason, the overall SIRS trial results (which focus primarily on non-kidney outcomes), rather than the results of this AKI protocol, should be the primary information used to inform decisions about whether or not intravenous methylprednisolone should be adopted as a standard of care in cardiac surgery with a cardiopulmonary bypass pump.

\section{CONCLUSIONS}

The sample of SIRS AKI substudy far exceeds the cumulative number of patients enrolled across 70 different randomised controlled trials, which tested strategies to prevent or treat AKI in cardiac surgery. ${ }^{23}$ Strengths of this protocol and the overall SIRS trial are generalisable estimates derived from patients recruited in 81 centres in 18 countries and rigorous randomised trial methodology (eg, concealed allocation, placebo-controlled trial, blinded central adjudication of the outcomes). In this report, we have judiciously prespecified the main questions and analytical protocol that will be used to test relevant AKI hypotheses in SIRS. We have done so to minimise the chance of spurious post hoc assertions of effect, so that the AKI results from this large international are robust and believable.

\author{
Author affiliations \\ ${ }^{1}$ Western University, London, Canada \\ ${ }^{2}$ Population Health Research Institute/McMaster University/Hamilton Health \\ Sciences, Hamilton, Canada \\ ${ }^{3}$ University of Waterloo, Waterloo, Canada \\ ${ }^{4}$ Yale University, Connecticut, USA \\ ${ }^{5}$ Department of Outcomes Research, Cleveland Clinic, Cleveland, Ohio, USA \\ ${ }^{6}$ West China Hospital/Sichuan University, Chengdu, China \\ ${ }^{7}$ Princess Alexandra Hospital, Woolloongabba, Australia \\ ${ }^{8}$ Tehran Heart Center, Tehran University of Medical Sciences, Tehran, Iran \\ ${ }^{9}$ Centre Hospitalier de l'Université de Montréal, Montréal, Canada \\ ${ }^{10}$ University of Thessaly, Larissa, Greece \\ ${ }^{11}$ Montreal Heart Institute, Montréal, Canada \\ ${ }^{12}$ The Chinese University of Hong Kong, Hong Kong Special Administrative \\ Region, China \\ ${ }^{13}$ Université de Sherbrooke, Sherbrooke, Canada
}

Acknowledgements The authors thank Dr Mitesh Shah for his contributions when submitting the associated Canadian Institutes of Health Research grant, which included a background literature review.

Funding The SIRS trial and AKI substudy are completely funded by the Canadian Institutes of Health Research (application number of the AKI substudy: 259720).

Competing interests None.

Ethics approval Ethics review boards at 81 centres in 18 countries.

Provenance and peer review Not commissioned; peer reviewed for ethical and funding approval prior to submission.

Data sharing statement No additional data are available.

Open Access This is an Open Access article distributed in accordance with the Creative Commons Attribution Non Commercial (CC BY-NC 3.0) license, which permits others to distribute, remix, adapt, build upon this work noncommercially, and license their derivative works on different terms, provided the original work is properly cited and the use is non-commercial. See: http:// creativecommons.org/licenses/by-nc/3.0/

\section{REFERENCES}

1. Chertow GM, Burdick E, Honour M, et al. Acute kidney injury, mortality, length of stay, and costs in hospitalized patients. J Am Soc Nephrol 2005;16:3365-70.

2. Ricci Z, Cruz D, Ronco C. The RIFLE criteria and mortality in acute kidney injury: a systematic review. Kidney Int 2008;73:538-46.

3. Coca SG, Yusuf B, Shlipak MG, et al. Long-term risk of mortality and other adverse outcomes after acute kidney injury: a systematic review and meta-analysis. Am J Kidney Dis 2009;53:961-73.

4. Rosner MH, Okusa MD. Acute kidney injury associated with cardiac surgery. Clin J Am Soc Nephrol 2006;1:19-32.

5. Bronicki RA, Backer CL, Baden HP, et al. Dexamethasone reduces the inflammatory response to cardiopulmonary bypass in children. Ann Thorac Surg 2000;69:1490-5.

6. Yared JP, Starr NJ, Torres FK, et al. Effects of single dose, postinduction dexamethasone on recovery after cardiac surgery. Ann Thorac Surg 2000;69:1420-4.

7. Dieleman JM, Nierich AP, Rosseel PM, et al. Intraoperative high-dose dexamethasone for cardiac surgery: a randomized controlled trial. JAMA 2012;308:1761-7.

8. Whitlock RP, Chan S, Devereaux PJ, et al. Clinical benefit of steroid use in patients undergoing cardiopulmonary bypass: a meta-analysis of randomized trials. Eur Heart J 2008;29:2592-600.

9. Loef BG, Henning RH, Epema AH, et al. Effect of dexamethasone on perioperative renal function impairment during cardiac surgery with cardiopulmonary bypass. Br J Anaesth 2004;93:793-8.

10. Prasongsukarn K, Abel JG, Jamieson WR, et al. The effects of steroids on the occurrence of postoperative atrial fibrillation after coronary artery bypass grafting surgery: a prospective randomized trial. J Thorac Cardiovasc Surg 2005;130:93-8.

11. Whitlock RP. http://clinicaltrials.gov/show/NCT00427388. 2014 (accessed 16 Jan 2014)

12. Kidney Disease: Improving Global Outcomes (KDIGO) Acute Kidney Injury Work Group. KDIGO clinical practice guidelines for acute kidney injury. Kidney Int 2012;2:1-138.

13. Levey AS, Stevens LA, Schmid CH, et al. A new equation to estimate glomerular filtration rate. Ann Intern Med 2009;150:604-12.

14. Austin PC. Absolute risk reductions, relative risks, relative risk reductions, and numbers needed to treat can be obtained from a logistic regression model. J Clin Epidemiol 2010;63:2-6.

15. White IR, Thompson SG. Adjusting for partially missing baseline measurements in randomized trials. Stat Med 2005;24:993-1007.

16. Altman DG. Confidence intervals for the number needed to treat. BMJ 1998;317:1309-12.

17. Bender R. Calculating confidence intervals for the number needed to treat. Control Clin Trials 2001;22:102-10.

18. Pogue J, Devereaux PJ, Thabane L, et al. Designing and analyzing clinical trials with composite outcomes: consideration of possible treatment differences between the individual outcomes. PLOS ONE 2012;7:e34785

19. SAS. http://support.sas.com/documentation/cdl/en/etsug/60372/ HTML/default/viewer.htm\#etsug_autoreg_sect024.htm, 2014 (accessed 16 Jan 2014).

20. Brown JR, Kramer RS, Coca SG, et al. Duration of acute kidney injury impacts long-term survival after cardiac surgery. Ann Thorac Surg 2010;90:1142-8.

21. Lems WF, Gerrits MI, Jacobs JW, et al. Changes in (markers of) bone metabolism during high dose corticosteroid pulse treatment in patients with rheumatoid arthritis. Ann Rheum Dis 1996;55:288-93.

22. Gaspari F, Ruggenenti P, Porrini E, et al. The GFR and GFR decline cannot be accurately estimated in type 2 diabetics. Kidney Int 2013;84:164-73.

23. Park M, Coca SG, Nigwekar SU, et al. Prevention and treatment of acute kidney injury in patients undergoing cardiac surgery: a systematic review. Am J Nephrol 2010;31:408-18. 